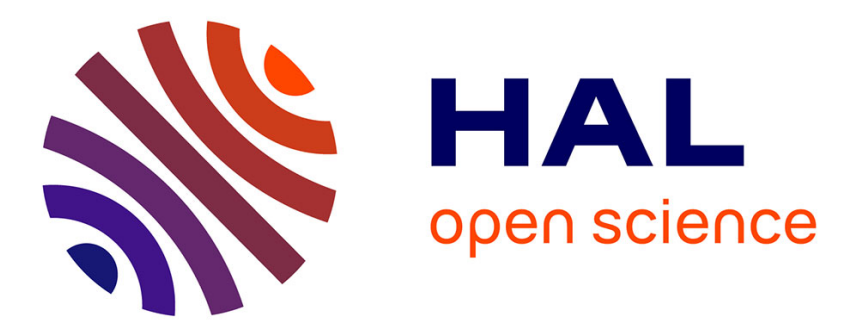

\title{
Analysis of a Multicarrier Communication System Based on Overcomplete Gabor Frames
}

\author{
Alexandre Marquet, Cyrille Siclet, Damien Roque, Pierre Siohan
}

\section{To cite this version:}

Alexandre Marquet, Cyrille Siclet, Damien Roque, Pierre Siohan. Analysis of a Multicarrier Communication System Based on Overcomplete Gabor Frames. Dominique Noguet; Klaus Moessner; Jacques Palicot. Cognitive Radio Oriented Wireless Networks, 172, Springer International Publishing, pp.387, 2016, Lecture Notes of the Institute for Computer Sciences, Social Informatics and Telecommunications Engineering, 978-3-319-40352-6. 10.1007/978-3-319-40352-6_32 . hal-01338564

\section{HAL Id: hal-01338564 https://hal.science/hal-01338564}

Submitted on 28 Jun 2016

HAL is a multi-disciplinary open access archive for the deposit and dissemination of scientific research documents, whether they are published or not. The documents may come from teaching and research institutions in France or abroad, or from public or private research centers.
L'archive ouverte pluridisciplinaire HAL, est destinée au dépôt et à la diffusion de documents scientifiques de niveau recherche, publiés ou non, émanant des établissements d'enseignement et de recherche français ou étrangers, des laboratoires publics ou privés. 


\title{
Analysis of a Multicarrier Communication System Based on Overcomplete Gabor Frames
}

\author{
Alexandre Marquet ${ }^{1,2}$, Cyrille Siclet ${ }^{1}$, Damien Roque $^{2}$, and Pierre Siohan ${ }^{3}$ \\ 1 Univ. Grenoble Alpes, GIPSA-Lab, F-38400 Grenoble, France. \\ alexandre.marquet@gipsa-lab.grenoble-inp.fr \\ 2 Institut Supérieur de l'Aéronautique et de l'Espace (ISAE-SUPAERO), Univ. \\ Toulouse, F-31055 Toulouse, France. \\ 3 (Retired from) Orange-Labs, F-35512 Cesson-Sevigné, France.
}

\begin{abstract}
A multicarrier signal can be seen as a Gabor family whose coefficients are the symbols to be transmitted and whose generators are the time-frequency shifted pulse shapes to be used. In this article, we consider the case where the signaling density is increased such that interpulse interference is unavoidable.

Such an interference is minimized when the Gabor family used is a tight frame. We show that, in this case, interference can be approximated as an additive Gaussian noise. This allows us to compute theoretical and simulated bit-error-probability for a non-coded system using a quadrature phase-shift keying constellation. Such a characterization is then used in order to predict the convergence of a coded system using low-density parity check codes. We also study the robustness of such a system to errors on the received bits in an interference cancellation context.
\end{abstract}

Key words: multicarrier modulations, faster-than-Nyquist signaling, linear system, optimal pulse-shapes, Gabor frames, interference analysis, interference cancellation, low-density parity check codes.

\section{Introduction}

In most of current communication systems, the linear part allows for perfect symbol reconstruction: the synthesis and analysis families used in the transmitter and the receiver form biorthogonal frames (also known as Riesz bases). In a single-carrier bandlimited scenario, this requires the Nyquist criterion to be respected [7]. In other words, the transmission rate must be lower than the bilateral bandwidth of the transmitted signal.

With an increasing need of spectral efficiency driven by overcrowded frequency bands, the main strategy relies on an increase of constellation size while keeping a constant transmission power, bandwidth and symbol rate (below the Nyquist limit). This choice induces a decrease of the distance between symbols, and the transmitted signal becomes more sensitive to noise, thus increasing biterror-probability [5].

A more unusual way to improve spectral efficiency is to increase the symbol rate until the Nyquist criterion is overridden, leading to unavoidable inter-pulse 
interference (IPI). This idea has been proposed by J. Mazo under the denomination "faster-than-Nyquist" (FTN) [6]. He showed that an increase up to approximately $25 \%$ of the Nyquist symbol rate keeps the minimal distance between symbols unchanged. As a consequence, considering the work of G.D. Forney on the optimal detection in presence of inter-symbol interference, one can preserve an acceptable bit-error-probability at the price of a greater computational complexity at the receiver side [5] (e.g.: maximum likelihood approaches...).

FTN transmission techniques can be extended to multicarrier modulations [9]. In this case, denoting $F_{0}$ the inter-carrier spacing and $T_{0}$ the multicarrier symbol duration, it can be shown that if $\rho=1 /\left(F_{0} T_{0}\right)>1$ then the synthesis and analysis families, respectively used for transmission and reception, can no longer be biorthogonal but can still form overcomplete frames [3]. This leads to IPI both in time and/or frequency. Numerous studies focus on the realization of coded multicarrier FTN systems using, in particular, series or parallel concatenations [10] as well as turboequalization techniques [4]. Studies of these latter systems over additive white Gaussian noise (AWGN) channels show great performance, confirming their relevance, even if their intrinsic complexity makes their design and performance comparison particularly demanding in terms of simulation time.

In this article, we study a linear multicarrier system operating with overcomplete Gabor frames (i.e.: a generalization of an FTN system), as it plays a fundamental role in practical systems, including decision feedback and iterative structures (e.g.: turboequalizers). Our work includes guidelines for the design of such systems over an additive white Gaussian noise (AWGN) channel, only based on the linear part of the system. First of all, we focus on the determination of the expression of the bit-error-probability of our linear system provided that tight frames are used, as prescribed in [11] in order to maximize the signal to interference plus noise ratio (SINR). Secondly, we investigate the behavior of interference cancellation receivers in this context. Finally, we show how the bit-error-probability closed-form expression of the linear system can be used to guide the design of more complex structures (including iterative receivers such as turboequalizers).

This article is constructed as follows. Part 2 details the input-output relations of the system in presence of noise, based on the frame theory. This theoretical framework allows for the determination of the SINR and the theoretical biterror-probability, based on the assumption of normality of the interference. Part 3 first aims for the study of the statistical properties of the interference in an empirical way, as to confirm the relevance of its Gaussian approximation. We then present bit-error-rate (BER) simulations aiming at the verification of our theoretical results, and an example of how our closed-form expression of the error probability can predict the performance of a coded system is presented through the simulation of a non-linear system using low-density parity check (LDPC) codes. The last simulation scenario analyzes the relevance of interference cancellation techniques in this communication context. Finally, conclusions and insights are presented in section 4. 


\section{System model}

\subsection{Input-output relationship in presence of white Gausssian noise}

Let us denote $\boldsymbol{c}=\left\{c_{m, n}\right\}_{(m, n) \in \boldsymbol{\Lambda}} \in \ell_{2}(\boldsymbol{\Lambda})$ with $\boldsymbol{\Lambda} \subset \mathbf{Z}^{2}$, a sequence of zeromean, independent and identically distributed coefficients. Its variance is $\sigma_{c}^{2}$. The multicarrier signal is then written as:

$$
s(t)=\sum_{(m, n) \in \mathbf{\Lambda}} c_{m, n} g_{m, n}(t), \quad t \in \mathbf{R}
$$

with $\boldsymbol{g}=\left\{g_{m, n}\right\}_{(m, n) \in \mathbf{\Lambda}}$ a Gabor family, with parameters $F_{0}, T_{0}>0$ and whose elements are given by the generator filter (also known as prototype) $g(t) \in \mathcal{L}_{2}(\mathbf{R})$ such as:

$$
g_{m, n}(t)=g\left(t-n T_{0}\right) e^{j 2 \pi m F_{0} t} .
$$

As a result, the information carried by $c$ is regularly spread in the time-frequency plane (fig. 1) with a minimum distance $F_{0}$ in frequency and $T_{0}$ in time.

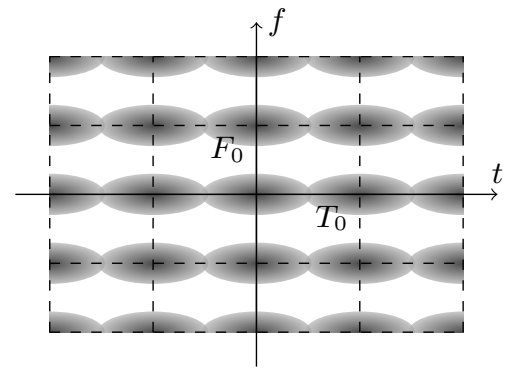

Fig. 1. Representation of a transmitted signal in the time-frequency plane. Here, the generator filter $g$ and the parameters of the lattice allow for a separation in the frequency domain, but not in the time domain.

In a real case scenario, we usually have $\boldsymbol{\Lambda}=\{0, \ldots, M-1\} \times\{0, \ldots, K-1\}$ where $M, K$ are strictly positive integers representing respectively the number of subcarriers and the number of multicarrier symbols to be transmitted. Such a restriction to a finite signaling set induces the convergence of the sum in (1). Nevertheless it can still contain a large amount of terms, so it is important to make it stable. Denoting $\mathcal{H}_{\boldsymbol{g}}=\overline{\operatorname{Vect}}(\boldsymbol{g})$ the closure of the linear span of the family $\boldsymbol{g}^{1}$, the stability of (1) is guaranteed when $\boldsymbol{g}$ is a Bessel sequence, which means that we can find an upper bound $B_{g}>0$ such as:

$$
\sum_{(m, n) \in \boldsymbol{\Lambda}}\left|\left\langle g_{m, n}, x\right\rangle\right|^{2} \leq B_{g}\|x\|^{2}, \quad \forall x \in \mathcal{H}_{\boldsymbol{g}}
$$

\footnotetext{
${ }^{1}$ The closure of a normed vector space $\mathbf{E}$ contains all the elements of $\mathbf{E}$, together with its limit elements. For example, the closure of the set of the rational numbers is the set of the real numbers.
} 
where $\langle\cdot, \cdot\rangle$ and $\|\cdot\|$ are the usual inner product and norm defined $\forall x, y \in \mathcal{L}_{2}(\mathbf{R})$ by

$$
\langle x, y\rangle=\int_{-\infty}^{+\infty} x^{*}(t) y(t) \mathrm{d} t, \quad\|x\|=\sqrt{\langle x, x\rangle}
$$

respectively, with $x^{*}$ the complex conjugate of $x$. In order to retrieve the data $c$ from the knowledge of $s(t)$, it is furthermore necessary (and sufficient) for $\boldsymbol{g}$ to be a linearly independent family. Hence $\boldsymbol{g}$ is a Riesz basis of $\mathcal{H}_{\boldsymbol{g}}$, in other words a family for which we can find $0<A_{g} \leq B_{g}$ such that:

$$
A_{g}\|x\|^{2} \leq \sum_{(m, n) \in \boldsymbol{\Lambda}}\left|\left\langle g_{m, n}, x\right\rangle\right|^{2} \leq B_{g}\|x\|^{2}, \forall x \in \mathcal{H}_{\boldsymbol{g}} .
$$

In this case, the density $\rho$ of $\boldsymbol{g}$ is necessarily lower than or equal to one: $\rho=$ $1 /\left(F_{0} T_{0}\right) \leq 1$. On the contrary, in order to increase the spectral efficiency of the system (for a fixed number of bits per symbol), this article focuses on the case where $\rho>1$. Thus, this increase in spectral efficiency is counterbalanced by an induced interference. In a linear receiver, this interference can be considered as an noise leading to an increased error probability. Indeed, when $\rho>1, \boldsymbol{g}$ is necessarily a linearly dependent Gabor family, but it may be an overcomplete frame of $\mathcal{L}_{2}(\mathbf{R})$, i.e. a family for which (5) is valid not only for $x \in \mathcal{H}_{\boldsymbol{g}}$, but for every $x \in \mathcal{L}_{2}(\mathbf{R})$. In this case, (1) is always stable and $\mathcal{H}_{\boldsymbol{g}}=\mathcal{L}_{2}(\mathbf{R})$. However, $\boldsymbol{g}$ cannot be a basis of $\mathcal{L}_{2}(\mathbf{R})$.

A linear receiver is considered as a first stage of a more complete FTN system (necessarily non-linear in order to yield acceptable performance). In this context, the estimated symbols $\hat{\boldsymbol{c}}=\left\{\hat{c}_{p, q}\right\}_{(p, q) \in \boldsymbol{\Lambda}}$ are given by

$$
\hat{c}_{p, q}=\left\langle\check{g}_{p, q}, r\right\rangle, \quad \forall(p, q) \in \boldsymbol{\Lambda}
$$

where $\check{\boldsymbol{g}}=\left\{\check{g}_{m, n}\right\}_{(m, n) \in \boldsymbol{\Lambda}}$ is a reception family, $r(t)=s(t)+n(t)$ is the signal seen by the receiver and $n(t)$ is a zero-mean white complex circular noise independent from the symbols, and whose bilateral power spectral density is $\gamma_{n}(f)=2 N_{0}$ for $f \in \mathbf{R}: \mathrm{E}(n(t))=0$ and $\mathrm{E}\left(n^{*}(t) n\left(t^{\prime}\right)\right)=2 N_{0} \delta\left(t-t^{\prime}\right)$, with $\mathrm{E}(\cdot)$ the expectation operator.

\subsection{Interference and noise analysis}

By rewriting (6), we can clearly identify the interference and noise terms:

$$
\hat{c}_{p, q}=\underbrace{c_{p, q}\left\langle\check{g}_{p, q}, g_{p, q}\right\rangle}_{\tilde{c}_{p, q}: \text { useful signal }}+\underbrace{\sum_{(m, n) \in \boldsymbol{\Lambda} \backslash\{(p, q)\}} c_{m, n}\left\langle\check{g}_{p, q}, g_{m, n}\right\rangle}_{i_{p, q}: \text { interference }}+\underbrace{\left\langle\check{g}_{p, q}, n\right\rangle}_{n_{p, q}: \text { :noise }} .
$$

We already showed in [11] that the SINR is maximized when $\check{\boldsymbol{g}}$ and $\boldsymbol{g}$ are dual canonical $\left(A_{g}=1 / A_{\check{g}}\right.$ and $\left.B_{g}=1 / B_{\check{g}}\right)$ tight $\left(A_{g}=B_{g}\right.$ and $\left.\check{\boldsymbol{g}}=\boldsymbol{g} / A_{g}\right)$ frames. This leads to the following expressions: 


$$
\begin{aligned}
E_{s} & =\frac{1}{2} \sigma_{c}^{2}\|g\|^{2}=\frac{\sigma_{c}^{2} A_{g}}{2 \rho} \\
\sigma_{i}^{2} & =\mathrm{E}\left(\left|i_{p, q}\right|^{2}\right)=(\rho-1) \sigma_{c}^{2} \\
\sigma_{n}^{2} & =\mathrm{E}\left(\left|n_{p, q}\right|^{2}\right)=2 \frac{\rho}{A_{g}} N_{0}
\end{aligned}
$$

with $E_{s}$ the per-symbol energy, $\sigma_{i}^{2}$ the variance of the interference and $\sigma_{n}^{2}$ the variance of the filtered noise. The SINR is then written as

$$
\mathrm{SINR}=\frac{1}{\rho-1+\frac{N_{0}}{E_{s}}} .
$$

We can see that the interference term $i_{p, q}$ is a random variable independent from the noise and corresponding to the sum of a large number of random variables $\tilde{c}_{m, n}$ which are zero-mean, independent, following the same type of law but with different variances $\sigma_{\tilde{c}_{m, n}}^{2}$ :

$$
\tilde{c}_{m, n}=c_{m, n}\left\langle\check{g}_{p, q}, g_{m, n}\right\rangle \text { and } \sigma_{\tilde{c}_{m, n}}^{2}=\sigma_{c}^{2}\left|\left\langle\check{g}, g_{m-p, n-q}\right\rangle\right|^{2} \text {. }
$$

All the conditions for applying the central limit theorem are thus not fulfilled but, as shown by our simulations in part 3.1, the Gaussian approximation is accurate for the sake of error-probability estimation. That is why in the following, we will assume the interference $i_{p, q}$ to be a normal zero-mean random variable independent from the noise. This is analogous to a case where the symbols would have been transmitted through an AWGN channel characterized by a signal-tonoise ratio given by (11). It is interesting to note that the noise term $n_{p, q}$ is zero-mean and Gaussian, but not necessarily white.

\subsection{Theoretical error probability}

We now restrict our analysis to the case where the symbols $c$ are taken from a quadrature phase-shift keying (QPSK) constellation. In that case, given the fact that both the noise and the interference are considered Gaussian, the bit error probability for a transmission through an AWGN channel is given by

$$
P_{e}=Q(\sqrt{\mathrm{SINR}})=Q\left(\sqrt{\frac{1}{(\rho-1)+\frac{N_{0}}{2 E_{b}}}}\right)
$$

where $Q(\cdot)$ is the complementary cumulative distribution function of a standard normal distribution and $E_{b}=E_{s} / 2$ the per-bit energy.

\section{Simulations}

\subsection{Empirical study of the interference term}

In this part, we discuss the relevance of the Gaussian approximation of the interference. To this extent, we measure $3.6 \times 10^{6}$ realizations of the interference 
$i_{p, q}$ term by performing a transmission of $M=64$ subcarriers over $K=50000$ multicarrier symbols for different values of $\rho$, using a QPSK constellation and tight frames. The variance of the obtained samples is then normalized thus giving standardized versions of $i_{p, q}$ depending on $\rho$, whose empirical probability density functions and cumulative distribution functions (CDF) are comparable. The behavior described here has been observed to be similar with both the real and the imaginary part of $i_{p, q}$, and for various prototypes forming tight frames.

Considering a transmission over a noise-free perfect channel (SINR $=1 /(\rho-$ 1)), zero-mean, independent and identically distributed bits, and denoting $F_{i, \rho}(x)$ the complementary CDF (CCDF) of the interference given a density of $\rho$, we can express the bit-error-probability as

$$
P_{e}(\rho)=1-F_{i, \rho}(\sqrt{\mathrm{SINR}})=1-F_{i, \rho}\left(\sqrt{\frac{1}{\rho-1}}\right) .
$$

In order to assess the Gaussian approximation, we compare the values of the functions $P_{e}(\rho)$ and $Q\left(\sqrt{\frac{1}{\rho-1}}\right)$ for various $\rho$ on figure 2. Even though the interference cannot be characterized by a Gaussian distribution, we can see that the relative approximation error is negligible, except for $\rho$ close to one, in this context of error probability estimation. Our simulations furthermore revealed that the Gaussian approximation then constitutes an upper bound for the biterror-probability. This result ensures that the Gaussian approximation can be safely used for multicarrier FTN communication system design and engineering, provided that tight frames are used.

\subsection{Linear system performance}

The simulations presented in this part consist in the transmission of $K=5000$ multicarrier symbols over $M=128$ subcarriers with a QPSK constellation. They were run for various prototypes. The prototypes maximizing the time-frequency localization (TFL) and minimizing the out-of-band energy (OBE) [8] form tight frames, as shown in [11] thanks to the Wexler-Raz theorem [3, theorem 9.3.4]. It is as well the case for the square-root-raised-cosine (SRRC) with roll-off factor $\alpha=\rho-1$ and the $T_{0}$-width rectangular $\left(\mathrm{RECT}_{T_{0}}\right)$ prototypes. When such a prototype is used both in transmission and reception, it is sufficient to set its norm to $1 / \sqrt{\rho}$ in order to obtain dual canonical tight frames with $A_{g}=1$. Although the rectangular prototype of width $\rho T_{0}$ and $T_{0}$ are able to form dual frames, they are not canonical dual. In addition, the pair of frames produced is non-tight so that using it in transmission and reception does not lead to a pair of canonical dual tight frames.

Figure 3 exhibits the perfect prediction of the SINR by (11) when the prototypes used in transmission and reception form a dual canonical tight pair of frames. In addition, figure 4 confirms the accuracy of the expression of the biterror-probability (13) and the relevance of the Gaussian approximation of the interference, although we can see its limits for strong $E_{b} / N_{0}(\geq 14 \mathrm{~dB})$ and $\rho$ close to $1(\rho=16 / 15)$. 


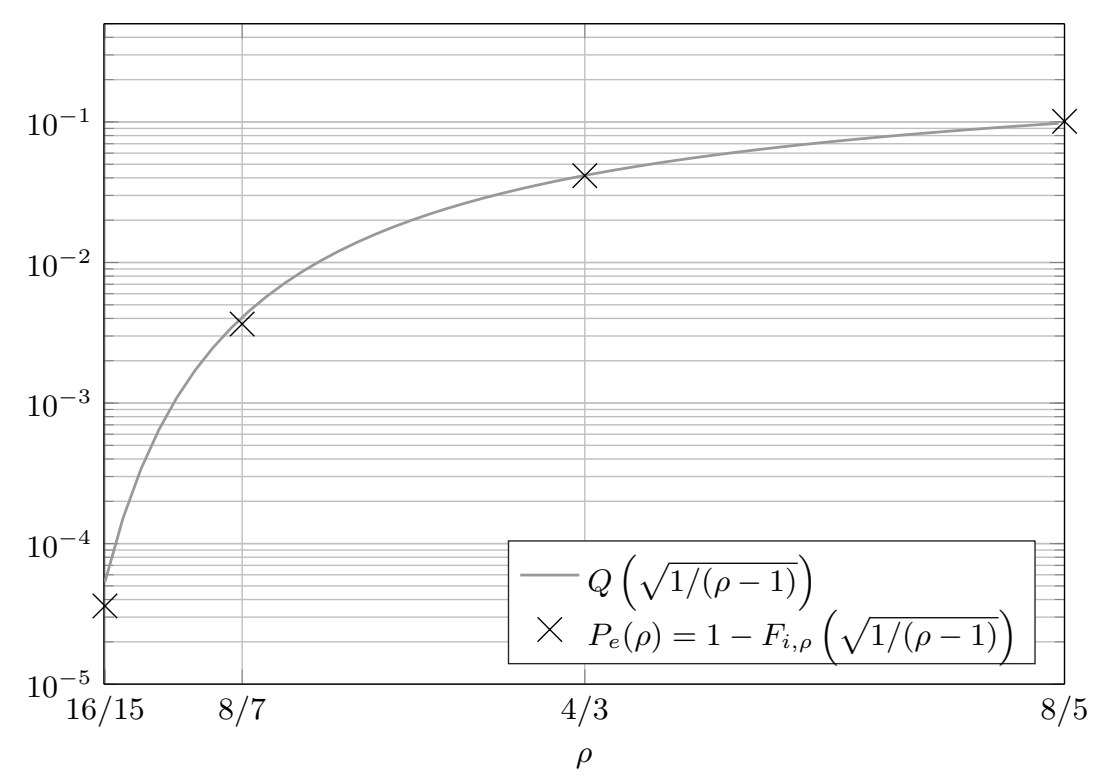

Fig. 2. Comparison of the real CCDF of the interference varying on $\rho$ and its Gaussian approximation.

In terms of performance, for this kind of non-coded multicarrier FTN system, figure 4 shows that the bit-error-rate (BER) rapidly rises with the density. We can also see on figure 5 that a lower-bound of the BER appears when the power of the noise becomes negligible compared to the one of the interference. In addition, and in accordance with the expression of the SINR, the performance gets worse if the frames used are not tight nor canonical dual. Theses results confirm the needs to develop non-linear detectors allowing for a more efficient IPI mitigation.

\subsection{Use in a coded system with iterative decoding}

BER curves of systems using efficient coding schemes such as turbocodes or LDPC are characterized by a so-called "convergence threshold" [2] which is the $E_{b} / N_{0}$ value from which the coded system achieves better performance than the uncoded one. Given an AWGN channel, it is also possible to characterize the coded system with a curve presenting the BER at the output of the decoder

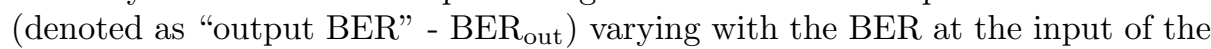

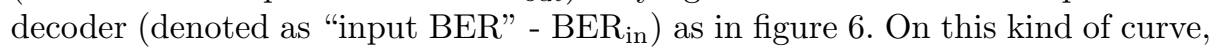
the convergence threshold is found at a given input BER. As a consequence, and thanks to the expression of the error probability (13), it is possible to determine the optimal density $\rho$ allowing the coded system to converge given an arbitrary value of $E_{b} / N_{0}$.

As an example, figure 6 shows that a coded system using the LDPC code of rate $1 / 2$ specified in the DVB-S2 specification [1] has its convergence threshold 
for an input BER of approximately 0.15. On figure 7, we can see that when used with a multicarrier FTN system using tight frames, the coded system converges as expected when the input BER goes below 0.15 , at $E_{b} / N_{0}=2 \mathrm{~dB}$.

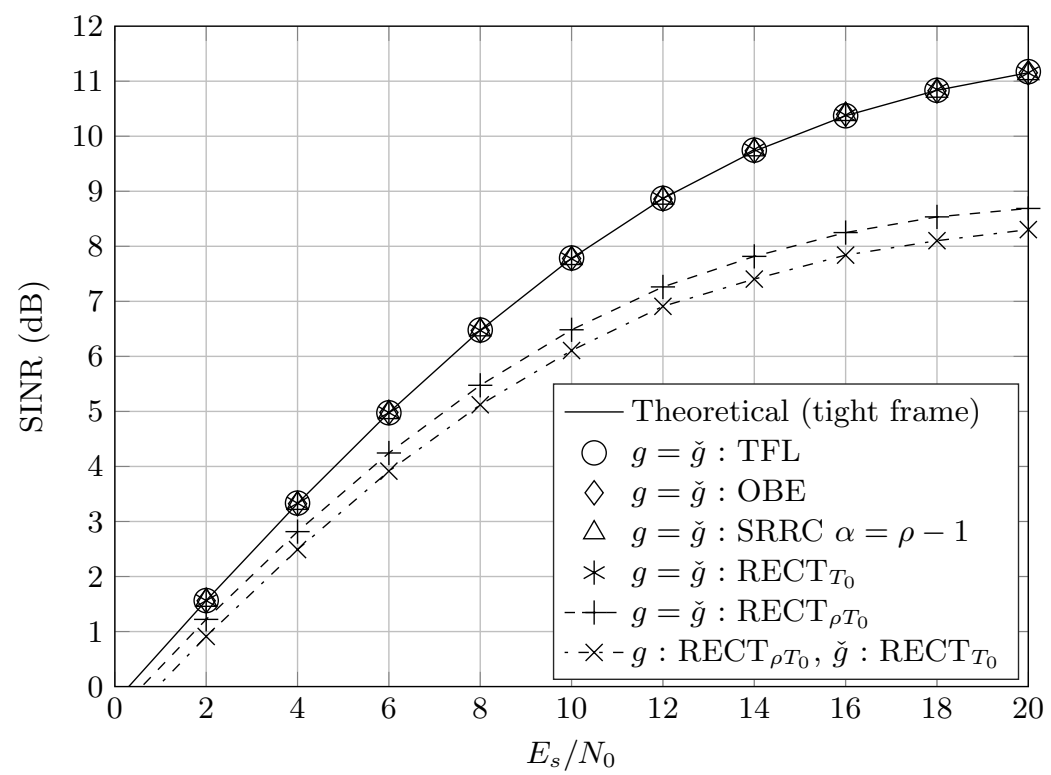

Fig. 3. SINR as a function of $E_{s} / N_{0}$, with $\rho=16 / 15$.

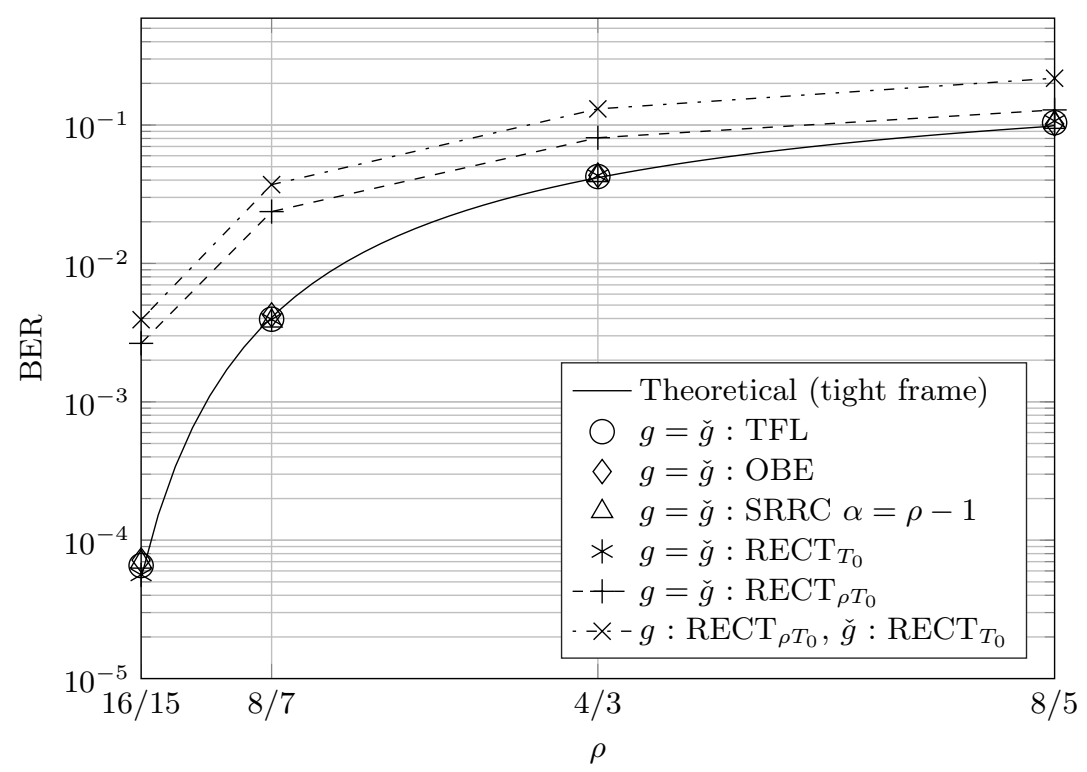

Fig. 4. BER as a function of $\rho$, with $E_{b} / N_{0}=20 \mathrm{~dB}$. 


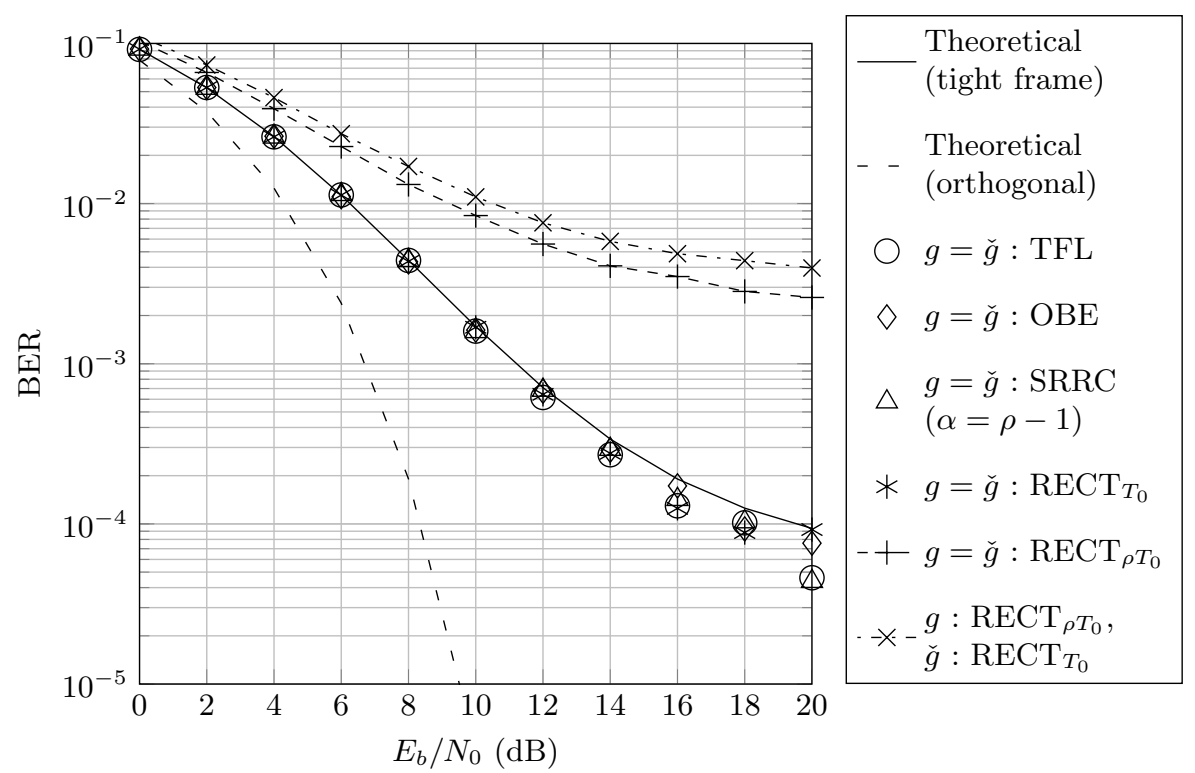

Fig. 5. BER as a function of $E_{b} / N_{0}$, with $\rho=16 / 15$.

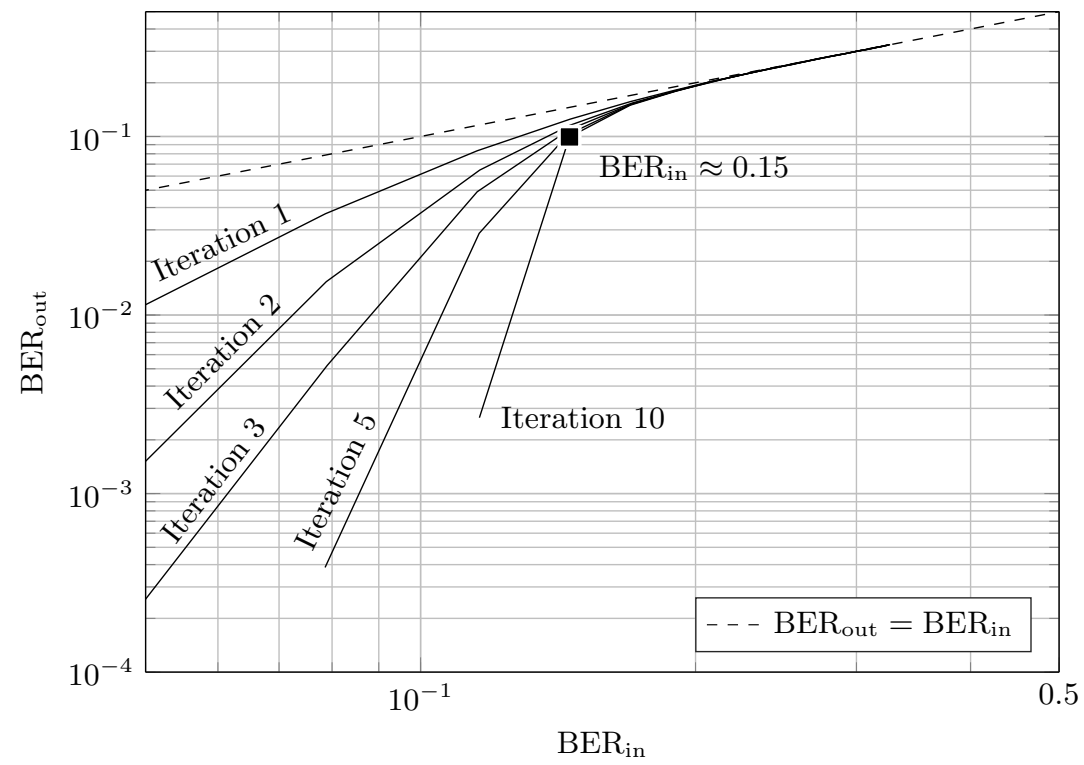

Fig. 6. Output BER as a function of the input BER for a rate $=1 / 2 \mathrm{LDPC}$ system. In this configuration, the convergence threshold is at an input BER of 0.15 .

\subsection{Performance with interference cancellation}

From the expression of the bit error probability (13) it is obvious that the FTN linear system shows worse performance compared to the orthogonal case. Be- 
sides, from the expression of the received signal (7), one can notice that the performance of the orthogonal system can be retrieved by removing the interference induced by the FTN system, allowing for an improvement of the spectral efficiency of the transmission while keeping the same BER.

Such an interference cancellation (IC) is usually performed by estimating the received symbols, then computing the interference term from these estimations and substract it to the received signal. Given that this estimation might not be perfect, it is interesting to assess the behavior of this system in presence of errors on the estimated symbols. To fulfill that purpose, we implemented the pseudogenie receiver depicted by figure 8 . The difference with a "true genie" receiver is that its knowledge of the transmitted symbols is corrupted by a binary symmetric channel inducing an error probability $P_{e, \text { genie }}$ on the bits used to compute the interference term.

Figure 9 presents the performance of this system, simulated by the transmission of $K=5000$ multicarrier symbols over $M=32$ subcarriers using a TFL prototype and a QPSK constellation. We can see that it is quite robust to the presence of errors on the bits used to compute and cancel the interference, which gives an insight on how non-linear receivers using interference cancellation (such as decision feedback or turboequalization) could efficiently prevent inter-carrier interference. Although not presented here, we ran simulations with other prototypes yielding tight frames, and obtained similar results.

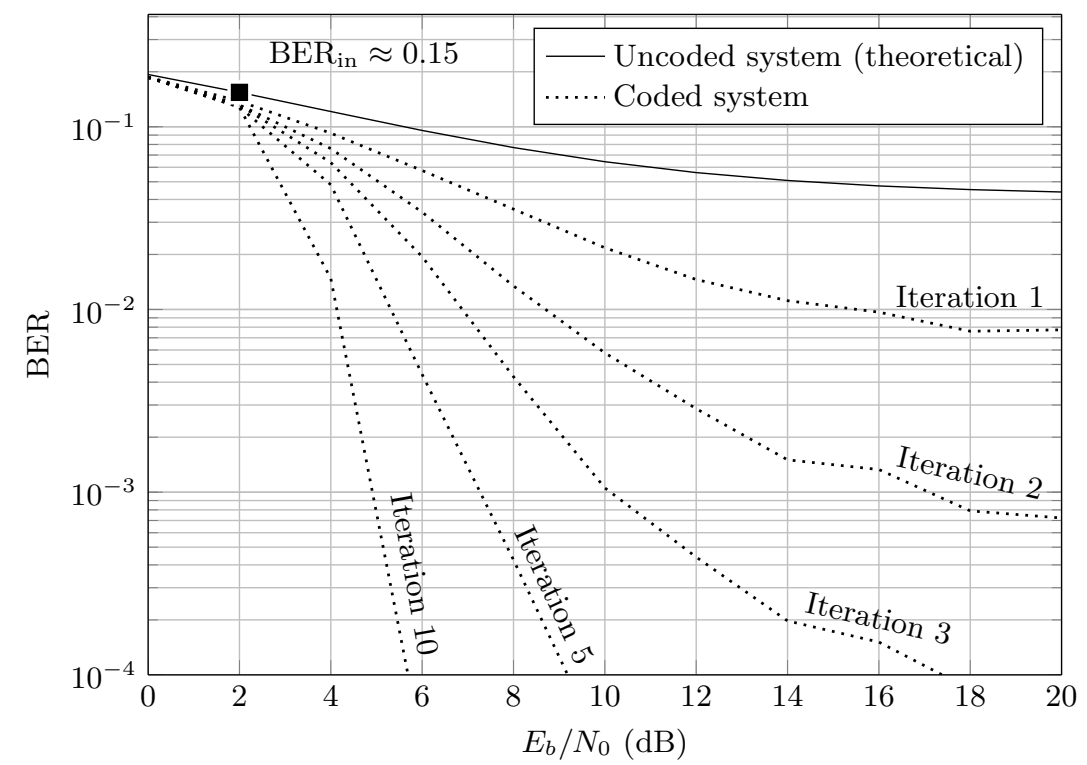

Fig. 7. BER as a function of $E_{b} / N_{0}$ using a rate $1 / 2$ LDPC code, $\rho=4 / 3$ and a TFL prototype for 10 iterations of the decoder. 


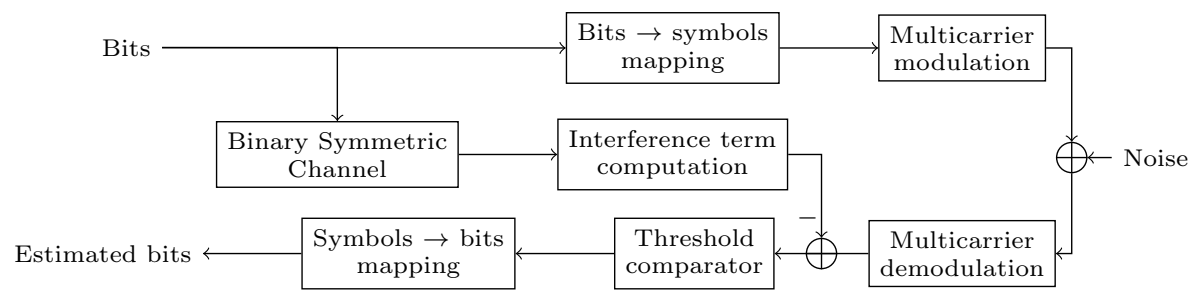

Fig. 8. Synoptic of the pseudo-genie IC receiver.

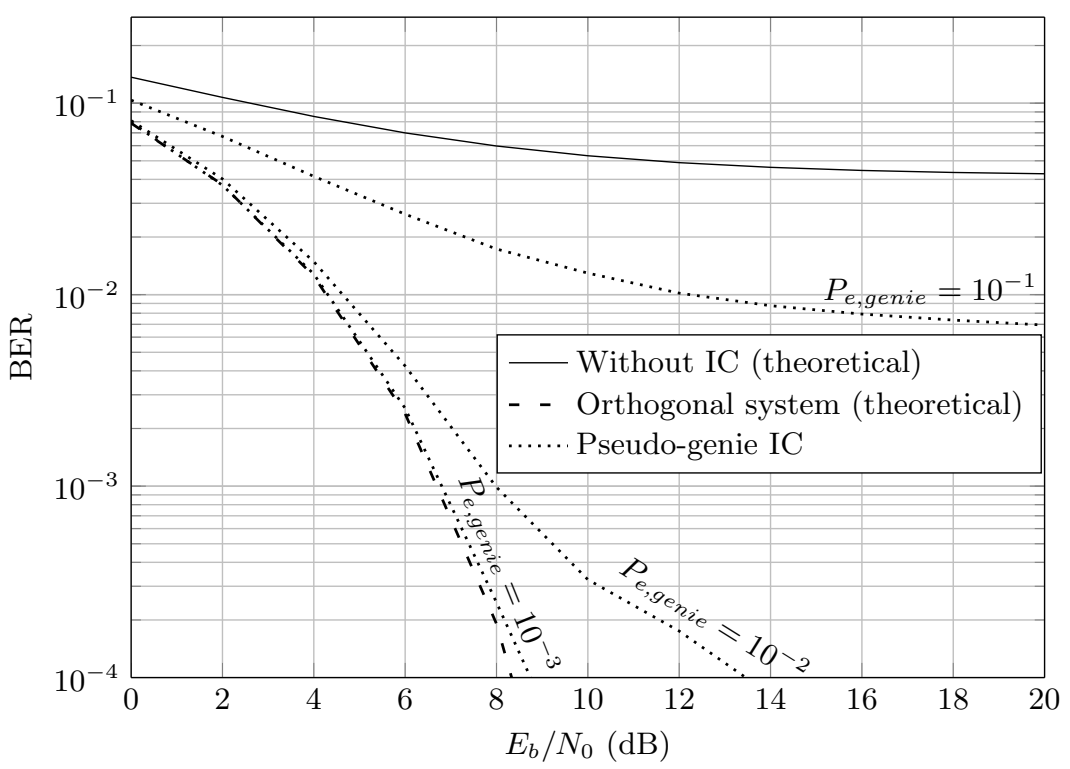

Fig. 9. BER as a function of $E_{b} / N_{0}$, with a pseudo-genie receiver, $\rho=4 / 3$, a TFL prototype and $P_{e, \text { genie }} \in\left\{10^{-1}, 10^{-2}, 10^{-3}\right\}$.

\section{Conclusion}

Through this article, we specified a linear multicarrier system based on the use of overcomplete Gabor frames, allowing an increase in signaling density in the time and/or the frequency domain and leading to a bidimensional FTN system. Consequently, an increase of the spectral efficiency beyond (bi)-orthogonal systems (for a given constellation size) yields interference between pulse-shapes. Such interference can be mitigated by the use of tight frames within the context of a linear system. Furthermore, we showed that interference cancellation based on noisy estimates of the transmitted symbols (pseudo-genie receiver) can lead to the same BER as orthogonal systems, but at a higher bitrate.

The results presented in this article allow the ability to relatively compare the performance of FTN multicarrier systems based on the parameters of their linear 
part (e.g.: time-frequency lattice density, transmission/reception filters...). Secondly, we showed how the knowledge of this performance can help the design of more complex receiver structures (e.g.: LDPC/turbodecoders, turboequalizers) by predicting their behavior.

Future work may consist in the analysis and efficient implementations of various multicarrier non-linear systems based on tight frames, and transmissions over more complex channels.

\section{Acknowledgment}

The authors would like to address a particular thank to Dr. Laurent Ros for his valuable advices and relevant remarks concerning this work.

\section{References}

1. ETSI: EN 302307 Digital Video Broadcasting (DVB); Second generation framing structure, channel coding and modulation systems for Broadcasting, Interactive services, News Gathering and other broadband satellite applications (DVB-S2) (2009)

2. Berrou, C., Amis Cavalec, K., Arzel, M., Glavieux, A., Jezequel, M., Langlais, C., Le Bidan, R., Saoudi, S., Battail, G., Boutillon, E., Saouter, Y., Maury, E., Laot, C., Kerouedan, S., Guilloud, F., Douillard, C.: Codes et turbocodes (sous la direction de Claude Berrou). Iris, Springer, Paris (2007)

3. Christensen, O.: Frames and bases: An introductory course. Birkhauser (2008)

4. Dasalukunte, D., Rusek, F., Owall, V.: Multicarrier faster-than-Nyquist transceivers: Hardware architecture and performance analysis. Circuits and Systems I: Regular Papers, IEEE Transactions on 58(4), 827 -838 (april 2011)

5. Forney, G.: Maximum-likelihood sequence estimation of digital sequences in the presence of intersymbol interference. Information Theory, IEEE Transactions on 18(3), 363-378 (1972)

6. Mazo, J.E.: Faster than Nyquist signaling. Bell System Technical Journal 54, 14511462 (October 1975)

7. Nyquist, H.: Certain topics in telegraph transmission theory. American Institute of Electrical Engineers, Transactions of the 47(2), 617-644 (1928)

8. Pinchon, D., Siohan, P.: Closed-form expressions of optimal short PR FMT prototype filters. In: Proc. IEEE Global Telecommunications Conf. GLOBECOM '11 (2011)

9. Rusek, F., Anderson, J.: The two dimensional Mazo limit. In: Information Theory, 2005. ISIT 2005. Proceedings. International Symposium on. pp. $970-974$ (sept 2005)

10. Rusek, F., Anderson, J.: Serial and parallel concatenations based on faster than Nyquist signaling. In: Information Theory, 2006 IEEE International Symposium on. pp. $1993-1997$ (july 2006)

11. Siclet, C., Roque, D., Shu, H., Siohan, P.: On the study of faster-than-Nyquist multicarrier signaling based on frame theory. In: Wireless Communications Systems (ISWCS), 2014 11th International Symposium on. pp. 251-255 (Aug 2014) 